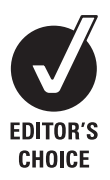

CHOICE

\title{
Increased incidence of sterile endophthalmitis following intravitreal preserved triamcinolone acetonide
}

\author{
J Jonisch, ${ }^{1}$ J C Lai, ${ }^{1,2}$ V A Deramo, ${ }^{1,2}$ A J Flug, ${ }^{1}$ D M Fastenberg ${ }^{1,2}$
}

${ }^{1}$ Department of Ophthalmology, North Shore-Long Island Jewish Health System, Great Neck, NY, USA; ${ }^{2}$ Long Island and Queens Vitreo-Retinal Consultants, Great Neck, NY, USA

Correspondence to: Dr J C Lai, 98-1079 Moanualua Rd, Suite 470, Aiea, HI 96701, USA; jimretina@yahoo.com

Accepted 25 April 2008

\section{ABSTRACT}

Aim: To report an increased incidence of sterile endophthalmitis following intravitreal injection of preserved triamcinolone acetonide (IVTA) from 1 May to 31 July 2006.

Methods: Charts were reviewed for all patients who underwent IVTA injections between 1 January 2005 and 31 July 2006 at the offices of a referral vitreo-retinal practice. Patients were included if they presented postIVTA with a clinical picture consistent with endophthalmitis.

Results: Between 1 January 2005 and 31 July 2006, 554 eyes underwent IVTA. Eleven eyes (1.9\%) developed an endophthalmitis. All eleven eyes underwent vitreous tap and intravitreal injection of antibiotics. All cultures and gram stains were negative for bacterial or fungal organisms. From 1 May to 31 July 2006, 97 eyes underwent IVTA. Nine eyes (9.3\%) developed sterile endophthalmitis. This represented a statistically significant $(p<0.0001)$ clustering of cases. Triamcinolone acetonide phials from affected lot numbers were analysed and were all found to be negative for bacterial endotoxin $(<0.05 \mathrm{EU} / \mathrm{ml})$.

Conclusion: Over the 19-month period analysed, 11 cases of sterile endophthalmitis occurred following IVTA, and nine of these cases were clustered over a 3-month period. No endotoxin was detected in the phials tested. The aetiology of this increased incidence of sterile endophthalmitis is still unclear.

Intravitreal injection of triamcinolone acetonide (IVTA) has been shown to be beneficial in the treatment of macular oedema secondary to vein occlusion, diabetes and uveitis, as well as adjunctive treatment of exudative macular degeneration. ${ }^{1-10}$ The most common side effects include ocular hypertension and cataract progression. ${ }^{11-14}$ Infectious endophthalmitis, pseudoendophthalmitis and sterile endophthalmitis are also well-known complications following IVTA. ${ }^{15-21}$ Pseudoendophthalmitis has been defined as cases where the injected agent, triamcinolone acetonide, creates the appearance of intraocular inflammation and/or hypopyon. ${ }^{14} 19$ Non-infectious or sterile endophthalmitis is defined as true intraocular inflammation following injection of IVTA not attributed to an infectious process. ${ }^{14}$ Historically, the commercially available form of triamcinolone acetonide used for intravitreal injection in the United States has been Kenalog (Bristol-Myers Squibb, Princeton, NJ) which comes in a preservative-containing formulation. The aetiology of sterile endophthalmitis is unknown, but several possible aetiologies have been proposed. It has been hypothesised that sterile endophthalmitis represents an inflammatory reaction to the triamcinolone acetonide, the preservatives in which it is suspended, or a form of bacterial endophthalmitis caused by contamination with bacterial toxins or endotoxin not detected by standard culture methods. ${ }^{1721}$ The reported incidence of sterile endophthalmitis ranges between $0.1 \%$ and $1.6 \%$ when using the preserved formulation. ${ }^{14}$ 16-18

In the months of May-July 2006, we observed an increased number of patients presenting with severe intraocular inflammation following injection of preserved IVTA. The purpose of this study is to analyse the incidence of sterile endophthalmitis following intravitreal injection of preserved triamcinolone acetonide from 1 January 2005 to 31 July 2006, to evaluate whether there was a statistically significant increased incidence.

\section{METHODS}

The North Shore-Long Island Jewish University Hospital System institutional review board approved the study protocol. Charts were reviewed for all patients who underwent intravitreal injection with preserved triamcinolone acetonide (Kenalog; Bristol-Myers Squibb, Princeton, NJ) between 1 January 2005 and 31 July 2006 at the offices of a referral vitreo-retinal practice. Charts were obtained by searching the computer database for all intravitreal injections performed, and included only those patients who underwent intravitreal injection with preserved triamcinolone acetonide. Records were reviewed of all patients who developed postinjection presumed endophthalmitis following intravitreal injection of preserved triamcinolone acetonide. Patients were included if they presented with a clinical picture consistent with endophthalmitis defined as postinjection vitritis, with fibrinoid anterior chamber reaction and/or hypopyon. Patients with pseudoendophthalmitis, with suspended triamcinolone crystals, were excluded.

Written informed consent was obtained from each patient prior to steroid injection. Standard aseptic techniques were used, and the procedure was performed in the office setting for all cases. The operative eye was given a preoperative topical antibiotic of the physician's choice. After the eye was anaesthetised with topical anaesthetic, a sterile lid speculum was placed in the eye. The temporal conjunctiva was then anaesthetised with a $4 \%$ lidocaine-hydrochloride soaked cotton tip. A $5 \%$ povidone-iodine solution was applied to the eye 
Table 1 Patient demographics

\begin{tabular}{|c|c|c|c|c|c|c|c|c|c|}
\hline $\begin{array}{l}\text { Patient with post-IVTA } \\
\text { endophthalmitis }\end{array}$ & Age & Indication & $\begin{array}{l}\text { Culture } \\
\text { results }\end{array}$ & Phakic status & $\begin{array}{l}\text { Capsule } \\
\text { status }\end{array}$ & $\begin{array}{l}\text { Pre-IVTA injection } \\
\text { visual acuity }\end{array}$ & $\begin{array}{l}\text { Presenting visual } \\
\text { acuity }\end{array}$ & $\begin{array}{l}\text { Visual acuity, } \\
1 \text { month }\end{array}$ & Month \\
\hline 1 & 62 & RVo & Negative & Pseudophakic & Intact & $20 / 100$ & HM & $20 / 80$ & May 2005 \\
\hline 2 & 76 & RVo & Negative & Phakic & NA & $20 / 80$ & $\mathrm{HM}$ & $20 / 60$ & Jan 2006 \\
\hline 4 & 67 & DME & Negative & Pseudophakic & Intact & $\mathrm{CF}$ & HM & $20 / 400$ & May 2006 \\
\hline 5 & 65 & DME & Negative & Phakic & NA & $20 / 200$ & $\mathrm{CF}$ & $20 / 150$ & May 2006 \\
\hline 6 & 76 & RVO & Negative & Pseudophakic & Ruptured & $20 / 60$ & HM & $20 / 100$ & June 2006 \\
\hline 9 & 81 & DME & Negative & Pseudophakic & Intact & $20 / 60$ & HM & $20 / 60$ & July 2006 \\
\hline 10 & 67 & CME & Negative & Pseudophakic & Intact & $20 / 70$ & HM & $20 / 70$ & July 2006 \\
\hline 11 & 85 & DME & Negative & Phakic & NA & $20 / 150$ & $H M$ & $20 / 60$ & July 2006 \\
\hline
\end{tabular}

$\mathrm{CF}$, count fingers; $\mathrm{CME}$, cystoid macular oedema secondary to pseudophakia; DME, diabetic macular oedema; HM, hand motion; IVTA, intravitreal injection of triamcinolone acetonide; NA, not applicable; RV0, retinal vein occlusion.

several minutes prior to intravitreal injection, as well as to the rubber stopper of the individual phials. The triamcinolone acetonide was prepared by drawing out $0.1 \mathrm{ml}$ from a $1 \mathrm{ml}$ single-use phial (Kenalog $40 \mathrm{mg} / \mathrm{ml}$ ) after vigorously shaking the suspension. The formulation was neither diluted nor changed, and the solvent was not removed. Intraocular pressure postinjection was measured. The injection protocol and the physicians performing the procedure were similar throughout the studied period. Patients were instructed to use a topical third- or fourth-generation flouroquinolone four times per day for 3 days postinjection.

Patient age, phakic status, status of capsular bag, any history of intraocular inflammation, indication for injection, month of injection and number of days to presentation with endophthalmitis were recorded for affected patients. Visual acuity was recorded on the day of IVTA injection, on day of presentation and at 1 month post-IVTA. The data were analysed using the Fisher exact test. Triamcinolone acetonide phials from three of the lot numbers affected were analysed for bacterial endotoxin using the limulus amebocyte assay. ${ }^{22}$

North Shore-Long Island Jewish Hospital's Department of Biostatistics was consulted to evaluate the significance of the cluster. The statistical question was whether the number of events (ie, cases of sterile endophthalmitis) follows a Poisson distribution. The Poisson distribution is frequently used in situations where events are rare, and one wishes to evaluate the significance of a cluster. The Poisson distribution assumes rare

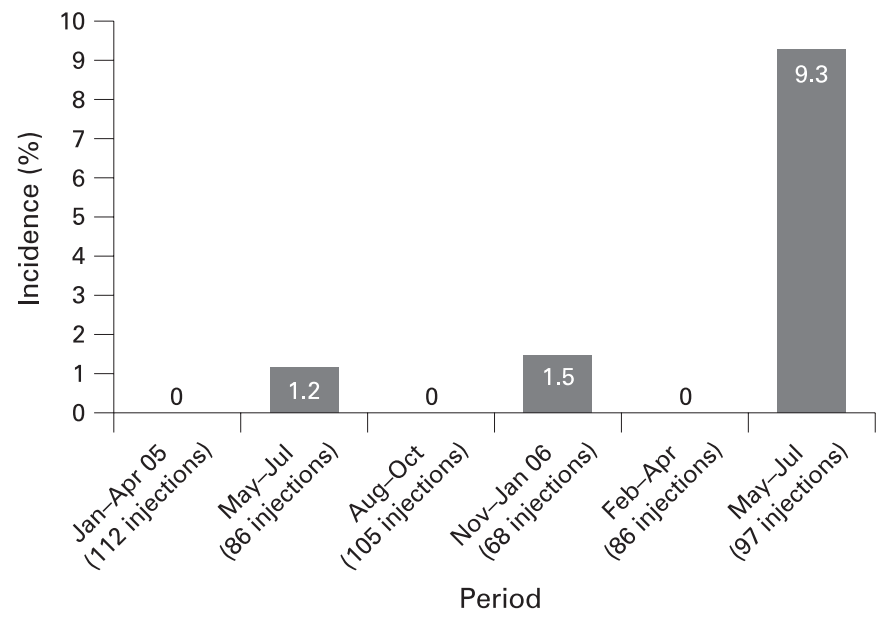

Figure 1 Incidence of sterile endophthalmitis. events occur at an average rate and independently of the time since the last event. A standard chi-square goodness of fit test was used to test the Poisson hypothesis, where the Poisson parameter, $\lambda$, was estimated as the total number of observed events/number of months in the study.

\section{RESULTS}

From 1 January 2005 through 31 July 2006, 554 eyes underwent IVTA. Eleven eyes (1.9\%) developed an endophthalmitis (table 1). Patient ages ranged from 62 to 85 . Indications for injection for these eyes included macula oedema secondary to vein occlusion $(n=4)$, diabetes $(n=4)$ and pseudophakia $(n=3)$. All patients presented by day 4 post-IVTA (average $=1.33$ days). Seven of 11 eyes were pseudophakic, and six of the seven pseudophakic eyes had intact posterior capsules. None of the 11 affected eyes had a prior history of intraocular inflammation. Pre-IVTA visual acuity ranged from 20/50 to hand motion. All patients with endophthalmitis presented with worsened visual acuity. Visual acuity at presentation with endophthalmitis was either hand motion or counting fingers vision. Visual acuity at 1 month post-IVTA injection ranged from 20/50 to 20/400. In nine of the 11 eyes affected, visual acuity at 1 month was equal to or better than the pre-IVTA injection visual acuity. All 11 eyes underwent vitreous tap and intravitreal injection of antibiotics with $1 \mathrm{mg}$ of vancomycin and $2.25 \mathrm{mg}$ of ceftazidime. All cultures and Gram stains were negative for bacterial or fungal organisms.

Between 1 May and 31 July 2006, 97 eyes underwent IVTA injections. Nine eyes (9.3\%) following the 97 injections developed a clinical picture of endophthalmitis.

Phials used from these cases were from varying lot numbers. Three phials from separate lot numbers affected were sent for endotoxin analysis using the limulus amebocyte assay, and each returned negative $(<0.05 \mathrm{EU} / \mathrm{ml})$ for bacterial endotoxin.

From 1 May to 31 July 2005, 86 eyes underwent IVTA injections. One eye (1.1\%) underwent a vitreous tap and intravitreal injection of antibiotics for endophthalmitis. In 2006, there were three cases in May, two cases in June and four cases in July. In 2005, there was one case in May and no cases in June or July. In the same 3-month time period the incidence rose from $1.1 \%$ to $9.7 \%(p=0.02)$ from 2005 to 2006 . No patient in either group had culture-positive endophthalmitis. The number of cases and the total number of IVTA injections for each 3month period studied are shown in fig 1. Beginning in August 2006, we began using a preservative-free formulation of triamcinolone acetonide. 
To evaluate the statistical significance of this cluster of cases, a standard chi-square goodness-of-fit (GOF) test was used to test the whether the number of cases of a rare event occur in a Poisson distribution. The Poisson distribution assumes that rare events occur at an average rate and independently of the time since the last event. The parameter, $\lambda$, was estimated as the total number of observed events/number of months in the study. The estimated mean for the Poisson distribution was $\lambda=11 / 19=0.58$ events/month. The GOF test showed a significant lack of fit for the Poisson distribution $(p<0.0001)$. The data were not consistent with the Poisson distribution assumption and support the hypothesis of a true cluster.

\section{DISCUSSION}

Preserved intravitreal triamcinolone acetonide has been reported to be therapeutically useful in cases of macular oedema due to multiple aetiologies and adjunctively in exudative macular degeneration. ${ }^{1-10}$ Infectious and non-infectious endophthalmitis remain important clinical entities. The reported incidence of sterile endophthalmitis following intravitreal injection of preserved triamcinolone acetonide ranges from $0.1 \%$ to $1.6 \%{ }^{16-18}$ Our observed incidence of $9.7 \%$ from 1 May to 31 July 2006 represents a statistically significant increased incidence when compared with the same time period 1 year earlier, and was a dramatic increase from our prior experience with Kenalog.

The reported incidence varies throughout the literature partly due to the variability in terminology used. We reserve the term sterile endophthalmitis, in the post-IVTA setting, to include a severe intraocular inflammation, which includes vitritis with an anterior chamber fibrinoid reaction and/or hypopyon not directly attributed to an infectious aetiology. The term pseudoendophthalmitis, in the post-IVTA setting, is reserved for cases in which the triamcinolone crystals appear in the anterior chamber with minimal associated inflammation.

Nelson et al reported seven cases of noninfectious endophthalmitis following 440 intravitreal Kenalog injections $(1.6 \%)$, although included in that number was at least one patient with only steroid particles within the anterior chamber 30 min postinjection. ${ }^{17}$ The actual incidence of non-infectious endophthalmitis was likely lower when eliminating the cases of pseudoendophthalmitis. Sutter and Gillies reported four cases following approximately 600 injections (0.6\%) that were described by the authors as pseudo-endophthalmitis, though each case presented with a dense vitreous haze. ${ }^{20}$ In a study by Roth et al, the incidence of sterile endophthalmitis was noted to be much higher (6.7\%, seven cases out of 104 injections) during a 14-month period between 1 May 2001 and 30 June 2002. ${ }^{21}$

The aetiology of sterile endophthalmitis is unclear. It has been postulated that sterile endophthalmitis may represent an atypical form of bacterial endophthalmitis caused by bacterial toxins or endotoxins not detected by standard culture methods. ${ }^{17}$ However, in our series, the triamcinolone acetonide was sent for analysis, and no endotoxin was detected. To our knowledge, this is the first reported formal analysis looking for endotoxin contamination of the triamcinolone.

Another theory is that sterile endophthalmitis represents an inflammatory reaction to the preservatives and additives contained in the Kenalog phials which includes benyzl alcohol, carboxymethylcellulose sodium, and polysorbate 80 in suspension. ${ }^{17}{ }^{21}$ This hypothesis is supported by the work of Jonas et al, who report an incidence of $0 \%$ in 454 eyes when the solvent was removed from triamcinolone acetonide. ${ }^{6}$ However, the recent reports of sterile endophthalmitis following intravitreal injection of preservative free triamcinolone acetonide would suggest that there is a yet-to-be-identified aetiology for this severe postinjection inflammation. ${ }^{23}$

There does not appear to be a good explanation for the statistically significant spike in the number of cases of sterile endophthalmitis that was witnessed in our practice during the spring of 2006. It is unknown how many other ophthalmologists experienced a similar increased incidence in this time period. There have been anecdotal reports of a similar rise in the number of cases of sterile endophthalmitis throughout parts of the country during the same time period (Eaton AM, personal communication). However, we are unaware of any published reports of this trend in a peer reviewed journal.

The development of complications following the off-label usage of a drug is particularly worrisome, particularly in light of the letter issued by the manufacturer of Kenalog (November 2006 letter to healthcare providers from Bristol-Myers Squibb Company, Princeton, NJ) (Lewis-Hall F, written communication, 22 November 2006). Reporting on the side-effect profile of medications becomes increasingly important with off-label usage, and the importance of the peer-review process cannot be overstated in these instances. As demonstrated by the recall of contact lens solution following the outbreak of Fusaruim keratitis, postmarketing vigilance is critical in detecting alarming trends that could alter currently accepted practice patterns. ${ }^{24}$ Specifically in this case, the spike in sterile endophthalmitis was the impetus for our decision to switch to preservative-free triamcinolone acetonide in an attempt to minimise the incidence of sterile endophthalmitis.

The side-effect profile of triamcinolone acetonide with and without preservatives needs further study. Recently the United States Food and Drug Administration (FDA) has approved a preservative-free formulation for intraocular use (Triesence; Alcon Laboratoy, Fort Worth, TX). Furthermore, a new formulation of triamcinolone acetonide designed for intraocular use is currently being examined in national clinical trials for macular oedema secondary to diabetes and vein occlusions. ${ }^{25}$ Until we gain a better understanding for the cause of sterile endophthalmitis, caution should be advised for the use of intravitreal Kenalog.

\section{Competing interests: None.}

Ethics approval: The North Shore-Long Island Jewish University Hospital System institutional review board approved the study protocol.

Patient consent: Obtained.

\section{REFERENCES}

1. Greenberg PB, Martidis $\mathrm{A}$, Rogers $\mathrm{AH}$, et al. Intravitreal triamcinolone acetonide for macular oedema due to central retinal vein occlusion. Br J Ophthalmol 2002;86:2478.

2. Martidis A, Duker JS, Greenberg PB, et al. Intravitreal triamcinolone for refractory diabetic macular edema. Ophthalmology 2002;109:920-7.

3. Jonas JB, Kreissig I, et al. Intravitreal injection of triamcinolone for diffuse diabetic macular edema. Arch Ophthalmol 2003;121:57-61.

4. Jonas JB, Sofker A. Intraocular injection of crystalline cortisone as adjunctive treatment of diabetic macular edema. Am J Ophthalmol 2001;132:425-7.

5. Antcliff RJ, Spalton DJ, Stanford MR, et al. Intravitreal triamcinolone for uveitic cystoid macular edema: an optical coherence tomography study. Ophthalmology 2001; 108:765-72.

6. Jonas JB, Kreissig I, Degenring RF. Intravitreal triamcinolone acetonide for pseudophakic cystoid macular edema. Am J Ophthalmol 2003;136:384-6.

7. Challa JK, Gillies MC, Penfold PL, et al. Exudative macular degeneration and intravitreal triamcinolone: 18-month follow-up. Aust N Z J Ophthalmol 1998; 26:277-81

8. Danis RP, Ciulla TA, et al. Intravitreal triamcinolone acetonide in exudative agerelated macular degeneration. Retina 2000;20:244-50.

9. Jonas JB, Kreissig I, Hugger $\mathbf{P}$, et al. Intravitreal triamcinolone acetonide for exudative age related macular degeneration. Br J Ophthalmol 2003;87:462-8. 
10. Spaide RF, Sorenson J, Maranan L. Photodynamic therapy with verteporfin combined with intravitreal injection of triamcinolone acetonide for choroidal neovascularization. Ophthalmology 2005;112:301-4.

11. Wingate RJ, Beaumont PE. Intravitreal triamcinolone and elevated intraocular pressure. Aust N Z J Ophthalmol 1999;27:431-2.

12. Bakri SJ, Beer PM. The effect of intravitreal triamcinolone acetonide on intraocular pressure. Ophthalmic Surg Lasers Imaging 2003;34:386-90.

13. Jonas JB, Kreissig I, Degenring R. Intraocular pressure after intravitreal injection of triamcinolone acetonide. Br J Ophthalmol 2003;87:24-7.

14. Jager RD, Aiello LP, et al. Risks of intravitreous injection: a comprehensive review. Retina 2004;24:676-98.

15. Jonas JB, Kreissig I, Degenring RF. Endophthalmitis after intravitreal injection of triamcinolone acetonide. Arch Ophthalmol 2003;121:1663-4.

16. Moshfeghi DM, Kaiser PK, Scott IU, et al. Acute endophthalmitis following intravitreal triamcinolone acetonide injection. Am J Ophthalmol 2003:136:791-6.

17. Nelson ML, Tennant MT, Sivalingam A, et al. Infectious and presumed noninfectious endophthalmitis after intravitreal triamcinolone acetonide injection. Retina 2003:23:686-91.
18. Westfall AC, Osborn A, Kuhl D, et al. Acute endophthalmitis incidence: intravitreal triamcinolone, Arch Ophthalmol 2005;123:1075-7.

19. Moshfeghi AA, Scott IU, et al. Pseudohypopyon after intravitreal triamcinolone acetonide injection for cystoid macular edema. Am J Ophthalmol 2004:138:489-92.

20. Sutter FK, Gillies MC. Pseudo-endophthalmitis after intravitreal injection of triamcinolone. Br J Ophthalmol 2003;87:972-4.

21. Roth DB, Chieh J, Spirn MJ, et al. Noninfectious endophthalmitis associated with intravitreal triamcinolone injection. Arch Ophthalmol 2003;121:1279-82.

22. Pearson FC, Dubczak J, Weary M, et al. Detection of endotoxin in the plasma of patients with Gram-negative bacterial sepsis by the Limulus amoebocyte lysate assay. J Clin Microbiol 1985;21:865-8.

23. Lorenzo CJ, González BM, Pérez Fl. Sterile endophthalmitis after benzyl alcohol filtered triamcinolone acetonide injection. Arch Ophthalmol 2008;126:142-3.

24. Chang DC, Grant GB, O’Donnell K, et al. Multistate outbreak of Fusarium keratitis associated with use of a contact lens solution. JAMA 2006;296:953-63.

25. Bhavsar AR, Ip MS, Glassman AR, DRCRnet and the SCORE Study Groups. The risk of endophthalmitis following intravitreal triamcinolone injection in the DRCRnet and SCORE clinical trials. Am J Ophthalmol 2007:144:454-6.

\title{
Endocapsular phacoemulsification without hydrodissec- tion: an effective technique for cataract surgery following anterior capsular tear
}

\begin{abstract}
A radial tear of the anterior capsule can occur either as a primary tear-out during capsulorrhexis or as a secondary event during phaco. Endocapsular surgery in this situation is hazardous and the risk of posterior propagation of the tear is significantly increased by performing hydrodissection. We describe an alternative technique of endocapsular phacoemulsification without primary hydrodissection. The risk of tear propagation is reduced whilst at the same time autohydrodissection of the cortico-capsular connections occurs, enabling rotation and removal of the nucleus. This simple modification of conventional phacoemulsification reduces the risk of posterior extension in cases of radial anterior capsular tear and allows the relatively safe completion of endocapsular phacoemulsification.
\end{abstract}

\section{R I Angunawela, B Little}

Department of Ophthalmology, Royal Free Hospital, London, UK

Correspondence to: Mr B Little, Consultant Ophthalmologist, Royal Free Hospital, London NW3 20G, UK; brianlittle@blueyonder.co.uk

To view the full report and accompanying video please go to: http://bjo.bmj.com/cgi/content/full/92/8/1054/DC1

All videos from the BJO video report collection are available from:

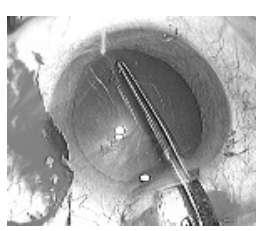

See online video reports

http://bjo.bmj.com/video/collection.dtl 